\title{
Un système génétique et pathologique complexe : le système phosphorylase-phosphorylase kinase
}

L'ensemble phosphorylase-phosphorylase kinase est responsable du premier temps de la dégradation du glycogène. Celle-ci utilise la voie de la phosphorolyse : le dernier glucose des chaînes latérales du glycogène est détaché sous forme de glucose-1phosphate. Les molécules de glucose sont enlevées une à une sous l'action de la phosphorylase; quand l'enzyme vient buter sur un point de branchement, celui-ci est scindé par une enzyme débranchante et le processus peut recommencer. Des données récentes ont redonné une actualité nouvelle à la physiologie et à la pathologie de cette phase initiale de la glycogénolyse.

La phosphorylase $(\mathrm{Ph})$ existe sous deux formes : une forme inactive $b$ et une forme active $a$, dérivant de la première par phosphorylation. La phosphorylase kinase (PhK) est l'enzyme qui catalyse cette phosphorylation. C'est dire que le couple $\mathrm{Ph}$ $\mathrm{PhK}$ est fonctionnellement indissociable. On comprend que la symptomatologie des déficits pose parfois des problèmes cliniques difficiles. En revanche, les mécanismes génétiques sont très différents pour les deux types de déficit.

Phosphorylase. La molécule de $\mathrm{Ph}$ est homogène, c'est-à-dire que les sous-unités qui la composent (deux, parfois quatre) sont identiques. Elle possède trois isozymes génétiquement différents. $\mathrm{La} \mathrm{Ph}$ musculaire est connue depuis plus de 50 ans $(\mathrm{C}$ et GT Cori). La séquence des 843 acides aminés de l'enzyme de lapin a été analysée dès 1977 ; son mécanisme d'activation requiert la phosphorylation d'une sérine en position 14. Son gène a été cloné en $\mathrm{m} / \mathrm{s} n^{\circ} 1$ vol. 6, janvier 90
1985 et localisé chez l'homme sur le chromosome 11 [1]. Le déficit en $\mathrm{Ph}$ musculaire est responsable de la maladie de McArdle ou glycogénose de type $\mathrm{V}$. Cette affection, à hérédité autosomique dominante, se manifeste typiquement par des crampes à l'effort, aboutissant à une myolyse parfois révélée par une myoglobinurie ; dans le muscle, indispensable au diagnostic puisqu'il est le seul tissu atteint, on ne trouve le plus souvent pas de protéine immunoréactive. Chez les huit malades dont l'ARN messager musculaire a été examiné, celui-ci était indétectable dans cinq cas, diminué en quantité mais de taille normale chez les trois autres. A notre connaissance, la lésion de l'ADN n'a pas encore été élucidée [2]. $\mathrm{La} \mathrm{Ph}$ hépatique a été beaucoup plus difficile à préparer que la forme musculaire. La séquence de ses acides aminés a été déduite de son ADNc. Chez l'homme, elle compte 845 acides aminés, avec une homologie de $80 \%$ vis-à-vis de celle du muscle. Le gène de la $\mathrm{Ph}$ du foie a été localisée sur le chromosome 14 [3]. Le déficit en phosphorylase hépatique (glycogénose de type VI), marqué par une hépatomégalie de l'enfance assez bien tolérée, avec absence de réponse glycogénolytique au glucagon, est authentique mais rare, le déficit en $\mathrm{PhK}$ hépatique qui le simule étant beaucoup plus fréquent. $\mathrm{La} \mathrm{Ph}$ cérébrale est de connaissance plus récente; outre le cerveau, elle est présente dans la plupart des tissus foetaux. Le clonage récent de son $\mathrm{ADNc}$, à partir d'une lignée d'astrocytome humain, a permis d'obtenir la séquence d'une protéine de 862 acides aminés, plus longue que celle des autres isozymes du fait d'une extension C-terminale. La comparaison avec les autres séquences la montre plus proche de la $\mathrm{Ph}$ musculaire que de la $\mathrm{Ph}$ hépatique. Le gène de la $\mathrm{Ph}$ cérébrale $\mathrm{a}$ été localisé sur le chromosome 20 , mais une homologie, due peut-être à un pseudogène, a été trouvée sur le chromosome 10 [4]. Aucun déficit en Ph cérébrale n'a été décrit, mais il est probable qu'aucune recherche n'a été dirigée dans cette voie.

Phosphorylase kinase. $\mathrm{La} \mathrm{PhK}$ forme un système beaucoup plus complexe que la Ph. Cette complexité est double. On connaît en effet des izozymes susceptibles de varier selon les tissus - et le dénombrement n'en est certainement pas achevé -, mais il existe surtout un échafaudage de quatre sous-unités différentes, dont le gène peut être porté par le chromosome $\mathrm{X}$ ou par un autosome; le déficit en une seule de ces sous-unités suffit à entraîner un déficit de l'ensemble par impossibilité de construire une molécule stable.

- L'édifice de la PhK. La PhK est formée de quatre sous-unités $\alpha, \beta, \gamma$, $\delta$, sous forme $(\alpha \beta \gamma \delta)^{4}$, donnant une masse moléculaire totale de $1,3 \times 10^{6}$ (Tableau I). La sous-unité $\delta$ est identique à la calmoduline, protéine ubiquitaire fixatrice du calcium. La sous-unité $\gamma$ porte l'activité catalytique, tandis que $\alpha$ et $\beta$ exercent des fonctions régulatrices à travers un système complexe de phosphorylations-déphosphorylations. Chacune des sous-unités a pu voir son gène localisé chez l'homme, tout au moins dans le cas de la PhK musculaire (Tableau I).

- Les isozymes de la PhK. Génétique et pathologie. L'existence d'iso- 
zymes de la PhK est certaine, bien qu'il reste difficile de les caractériser. Toute une série d'arguments, biochimiques et pathologiques, le suggèrent.

Arguments biochimiques d'abord: les PhK musculaire et hépatique présentent des différences notables dans leurs propriétés cinétiques. Lorsqu'on analyse les sous-unités en fonction de leur localisation tissulaire, la seule donnée certaine porte sur la sous-unité catalytique $\gamma$ : l'ADNc cloné à partir du muscle strié s'hybride à un messager correspondant présent dans le muscle et le cœur mais non dans le foie [5]; le gène de la chaîne $\gamma$ du foie est donc particulier et n'a pas encore été identifié. La chaîne $\alpha$ se présente dans le cœur sous une forme $\alpha$ ' légèrement plus petite, mais on ignore si cette différence signifie qu'il existe ou non deux gènes différents. Enfin, récemment a été réalisé le clonage des ADNc des deux grandes sous-unités $\alpha$ et $\beta$; il a permis de localiser les gènes correspondants [6] mais non, jusqu'à présent, de confirmer l'existence de plusieurs isozymes de ces sous-unités que suggèrent les données de la pathologie. Un fait impor- tant, sur lequel nous reviendrons, est qu'une de ces sous-unités, $\alpha$, a son gène porté par le chromosome $\mathrm{X}$.

En effet, de nombreuses maladies semblent relever du déficit en $\mathrm{PhK}$ et sont difficiles à raccorder entre elles. Chez l'homme, on peut distinguer trois et même peut-être quatre types. Le plus connu est le déficit en PhK hépatique responsable de la glycogénose de type VIII, de gravité modérée; impossible à distinguer cliniquement du déficit en $\mathrm{Ph}$ hépatique (type VI), elle est beaucoup plus fréquente. Le diagnostic peut se faire sur les cellules sanguines qui sont atteintes, mais le muscle est presque toujours indemne. Cette forme est transmise comme un caractère récessif lié au sexe. Une forme plus rare, à hérédité récessive autosomique, et qui touche à la fois muscle et foie, a été décrite en 1981, et une autre, purement musculaire, en 1980. Enfin, plus surprenant encore, un type purement cardiaque, comme l'a montré une élévation du glycogène exclusivement localisée au cœur, a fait l'objet de trois publications indépendantes.

Les modèles animaux ne sont pas moins déconcertants: le mieux connu est le mutant de la souche I, décrit en 1963 par Lyon et Porter. C'est une maladie récessive liée au sexe qui supprime à peu près complètement $(<0,2 \%)$ l'expression de la PhK du muscle sans affecter le foie; le taux dans le cœur est abaissé mais non nul. Le déficit n'est qu'incomplet à la naissance et se complète au cours du premier mois, ce qui suggère que la forme purement cardiaque diffère de la forme musculaire et pourrait être identique à une forme foetale [7]. Tout permet de penser que l'anomalie siège au niveau de la sous-unité $\alpha$, la seule dont le gène soit sur le chromosome $\mathrm{X}$, mais rien n'a encore été publié à ce sujet. On sait seulement que le messager de $\gamma$ est présent, mais ne peut donner naissance à une protéine stable. Curieusement, ces souris déficientes sont cliniquement presque normales. Une autre mutation de la souris, la souche $\mathrm{V}$, et transmise comme un caractère dominant lié à l'X, affecte tous les tissus et comporte la production d'une protéine anormale. Enfin un déficit localisé au foie, à hérédité autosomique récessive, a été décrit chez le rat.

On peut tenter, pour conclure, d'har-

Tableau I

NOMBRE D'ACIDES AMINÉS DES SOUS-UNITÉS ET LOCALISATION DES GĖNES DE Ph ET DE PhK

\begin{tabular}{|c|c|c|c|c|}
\hline & \multirow{2}{*}{$\begin{array}{l}\text { Nombre d'acides } \\
\text { aminés de la } \\
\text { sous-unité }\end{array}$} & \multirow{2}{*}{$\begin{array}{l}\text { Espèce } \\
\text { étudiée }\end{array}$} & \multicolumn{2}{|c|}{ Localisation chromosomique du gène } \\
\hline & & & Homme & Souris \\
\hline \multicolumn{5}{|l|}{ Phosphorylase } \\
\hline Muscle & 841 & Homme & $11 q 11-q 24$ & 19 \\
\hline Foie & 845 & Homme & $14 q 12-q 132$ & 12 \\
\hline Cerveau & 862 & Homme & 20 (homologie sur 10 ) & 2 \\
\hline \multicolumn{5}{|l|}{ Ph kinase } \\
\hline$\alpha$ & 1237 & Lapin & $X q 12-q 13$ & $\mathrm{x}$ \\
\hline$\beta$ & 1092 & Lapin & $16 q 12-q 13$ & \\
\hline$\gamma$ & 386 & Lapin & 7 (homologie sur 11 ) & \\
\hline$\dot{\delta}$ & 148 & Lapin & 7 et 14 (calmoduline) & \\
\hline
\end{tabular}


Tableau II

LISTE DES DÉFICITS CONNUS EN PHOSPHORYLASE KINASE ; ATTRIBUTION HYPOTHÉTIQUE DE L'ANOMALIE A UNE DES SOUS-UNNITÉS

\begin{tabular}{|c|c|c|c|c|}
\hline Espèce & $\begin{array}{l}\text { Nom de } \\
\text { la maladie }\end{array}$ & $\begin{array}{l}\text { Organe(s) } \\
\text { atteint(s) }\end{array}$ & Génétique & $\begin{array}{c}\text { Sous-unité } \\
\text { probablement en cause }\end{array}$ \\
\hline Homme & $\begin{array}{l}\text { (1) Glycogénose } \\
\text { type VIII } \\
\text { (2) } \\
\text { (3) } \\
\text { (4) }\end{array}$ & $\begin{array}{l}\text { Foie }+ \text { cellules sanguines } \\
\text { Tous organes } \\
\text { Muscle } \\
\text { Cœur seulement }\end{array}$ & $\begin{array}{l}\text { Récessif lié au sexe } \\
\text { Récessif autosomique } \\
\text { Récessif autosomique } \\
\text { Récessif autosomique }\end{array}$ & $\begin{array}{l}\alpha \text { foie } \\
\beta \\
\gamma \text { muscle } \\
\text { Isozyme cardiaque spéci- } \\
\text { fique ou forme fotale? }\end{array}$ \\
\hline $\begin{array}{l}\text { Souris } \\
\text { Souche I } \\
\text { Souche V } \\
\text { Rat }\end{array}$ & & $\begin{array}{l}\text { Muscle } \\
\text { Muscle } \\
\text { Foie }\end{array}$ & $\begin{array}{l}\text { Récessif lié au sexe } \\
\text { Dominant lié au sexe } \\
\text { Récessif autosomique }\end{array}$ & $\begin{array}{l}\alpha \text { muscle } \\
\alpha \text { muscle } \\
\gamma \text { foie }\end{array}$ \\
\hline
\end{tabular}

moniser les données déjà recueillies, ce qui permettra de souligner les recherches qui restent à faire et de se heurter à certaines contradictions. Laissant de côté la sous-unité $\delta$, car la calmoduline est ubiquitaire et on n'en connaît pas de déficits, on sait maintenant que deux sous-unités, $\beta$ et $\gamma$, sont portées par des autosomes, et une, $\alpha$, par l'X; cette dernière donnée, d'acquisition récente, permet de laisser de côté l'hypothèse qui faisait intervenir un ou des gènes régulateurs. Ainsi s'explique, en effet, l'existence de formes liées au sexe, les plus fréquentes. Un problème cependant demeure; les formes liées au sexe atteignent le muscle et épargnent le foie chez la souris I, alors que c'est l'inverse chez l'homme. Il existe probablement un locus spécifique de la sous-unité $\alpha$ du foie et une tâche prioritaire serait de le découvrir. Les formes récessives doivent être dues, lorsqu'elles sont autosomiques, à un déficit en $\beta$ ou $\gamma$. Comme nous l'avons vu, la sousunité catalytique $\gamma$ est différente dans le foie et le muscle, si bien que l'on peut légitimement faire l'hypothèse que si tous les tissus sont atteints, c'est $\beta$ qui est en cause; si le foie seul $\mathrm{m} / \mathrm{s} n^{\circ} 1$ vol. 6 , janvier 90 est atteint ou épargné, c'est probablement $\gamma$ (Tableau II). Reste la question troublante des atteintes cardiaques isolées; on peut postuler l'existence d'un isozyme cardiaque, peut-être identique à une forme fœtale, encore faudrait-il le démontrer.

Les maladies dues à un déficit en phosphorylase ou en phosphorylase kinase ne sont le plus souvent pas d'une haute gravité ; il est cependant important de les reconnaître car la surveillance des malades est nécessaire. Leur compréhension reste, dans bien des cas, un défi au biologiste, défi qui n'a encore été que partiellement relevé.

\section{Jean-Claude Dreyfus}

\section{RÉFÉRENCES}

1. Lebo RV, Gorin F, Fletterick R, et al. High resolution chromosome sorting and DNA spot-blot analysis assign McArdle's syndrome to chromosome II. Science 1984 ; 225 : 57-9.

2. Gautron S, Daegelen D, Mennecier F, Dubocq D, Kahn A, Dreyfus JC. Molecular mechanisms of McArdle's disease (muscle phosphorylase deficiency): RNA and DNA analysis. J Clin Invest 1987 ; 79 : 275-81.

3. Newgard CB, Fletterick RJ, Anderson LA Lebo RV. The polymorphic locus for glycogen storage disease VI (liver glycogen phosphorylase) maps to chromosome 14. Am J Hum Genet 1987 ; $40: 351-64$.

4. Newgard CB, Littman DR, Van Genderen C, Smith M, Fletterick RJ. Human brain phosphorylase. J Biol Chem 1988 ; 263 : 38507.

5. Bender PK, Emerson Jr CP. Skeletal mus cle phosphorylase kinase catalytic subunit mRNAs are expressed in heart but not in liver. J Biol Chem 1987; $262: 8799-805$.

6. Francke U, Darras BT, Zander MF, Kilimann MW. Assignment of human genes for phosphorylase kinase subunits (PHKA) to $\mathrm{Xql2}-\mathrm{q} 13$ an (PHKB) to $16 \mathrm{q} 12-\mathrm{ql}$. Am J Hum Genet 1989; 45 : 276-82.

7. Daegelen-Proux D, Alexandre Y, Dreyfus JC. Phosphorylase kinase isoenzymes in deficient ICR/IAM mice. Eur J Biochem $1978 ; 90: 369-75$

8. Human Gene Mapping 10. Cytogen Cell Genet $1989 ; 51$

9. Glaser T, Matthews KE, Hudson JW, et al. Localization of the muscle, liver and brain phosphorylase genes on linkage maps of mouse chromosomes 19,12 and 2 respectively. Genomics $1989 ; 5$ : 510-21. 